\title{
A REDUCTION THEORY FOR NON-SELF-ADJOINT OPERATOR ALGEBRAS
}

\author{
BY
}

\author{
E. A. $\left.\operatorname{AZOFF(}{ }^{1}\right)$, C. K. FONG( $\left.{ }^{2}\right)$ AND F. GILFEATHER
}

\begin{abstract}
It is shown that every strongly closed algebra of operators acting on a separable Hilbert space can be expressed as a direct integral of irreducible algebras. In particular, every reductive algebra is the direct integral of transitive algebras. This decomposition is used to study the relationship between the transitive and reductive algebra problems. The final section of the paper shows how to view direct integrals of algebras as measurable algebra-valued functions.
\end{abstract}

1. Introduction. Let $A$ be a Hilbert space operator commuting with an abelian von Neumann algebra $D$. Then von Neumann's reduction theory describes a method of decomposing $A$ with respect to $D$, i.e., a method of expressing $A$ as a direct integral of operators, $A=\int_{\Lambda}^{\oplus} A(\lambda) \mu(d \lambda)$, which strongly reflects the fact that $A$ is reduced by each projection in $D$. In a sense, the larger $D$ is, the simpler the direct integrands $\{A(\lambda)\}$ become; in particular, if $D$ is chosen to have a certain maximality property, then the $\{A(\lambda)\}$ turn out to be irreducible.

The main purpose of the present paper is to carry over von Neumann's decomposition to (not-necessarily-selfadjoint) algebras of operators. Specifically, we define a decomposition $\mathscr{U} \sim \int_{\Lambda}^{\oplus} \mathscr{A}(\lambda) \mu(d \lambda)$ for any algebra $\mathfrak{U}$ of operators commuting with $D$. The algebras $\{\mathscr{\mathscr { A }}(\lambda)\}$ are 'simpler' than the algebra $\mathscr{U}$-if $D$ is maximal, then almost all of the $\{\mathscr{U}(\lambda)\}$ are irreducible.

The motivation for our procedure stems from a desire to study the relationship between the transitive and reductive algebra problems. In fact, it was observed in [4] that a maximal decomposition of a reductive operator leads to transitive direct integrands-this allowed the authors of [4] to conclude that the transitive algebra problem for singly generated algebras (i.e., the invariant subspace problem) is equivalent to the reductive algebra problem for singly generated

Received by the editors February 14, 1975 and, in revised form, October 9, 1975. AMS (MOS) subject classifications (1970). Primary 46L15; Secondary 47A15, 28A05.

Key words and phrases. Direct integral of algebras, transitive algebra, reductive algebra, Borel structure.

(1) The first author acknowledges partial support under NSF grant GP 38488.

(2) The contribution of the second author represents part of his dissertation written under the direction of Professor Peter Rosenthal at the University of Toronto. The author wishes to thank Professor Rosenthal for his advice and encouragement. 
algebras (i.e., the reductive operator problem). In a similar fashion, the reduction theory of the preceding paragraph allows us to express every reductive algebra as a direct integral of transitive algebras. Although this decomposition does not imply the global equivalence of the transitive and reductive algebra problems, it does illuminate their relationship and allows us to state several results of the Dyer-Pederson-Porcelli type as corollaries, including a formulation of the transitive algebra problem in terms of the reductive algebra problem.

The structure of the remainder of the paper is as follows. $\$ 2$ is devoted to preliminaries, and includes a brief summary of parts of von Neumann's theory. The mechanics of the decomposition $\mathscr{U} \sim \int_{\Lambda}^{\oplus} \mathscr{U}(\lambda) \mu(d \lambda)$ are developed in $\S 3$ and the results are applied to reductive algebras in $\$ 4$.

The final section of the paper examines the way the direct integrands of a decomposable algebra 'fit together'. More specifically, we equip the collection of strongly-closed algebras with a certain natural Borel structure and show that for any decomposable $\mathscr{U} \sim \int_{\Lambda}^{\oplus} \mathscr{H}(\lambda) \mu(d \lambda)$, the algebras $\{\mathscr{A}(\lambda)\}$ depend measurably on $\lambda$. Parallel considerations for selfadjoint algebras were developed in [6] and $[10]$.

In closing this introductory section, it seems appropriate to emphasize the two basic distinctions between the present theory and von Neumann's decomposition of selfadjoint algebras into direct integrals of factors: (1) the algebra $D$ of diagonal operators need not be contained in $\mathfrak{U}$ and $(2)$ the algebra $\mathfrak{U}$ need not be selfadjoint. The difficulties occasioned by (1) can be summed up by saying that in passing from an algebra to its decomposition, some information is lost, i.e., different algebras may have the same decomposition; the analogous problem for selfadjoint decompositions was pointed out by Mautner [11]. Consequences of (2) include the facts that we have no 'double-commutant theorem' or 'Kaplansky density theorem' at our disposal. In their place, we exploit the relationship between algebras of operators and their lattices of invariant subspaces; this represents the main distinction between the present paper and any of the cited parallel work on selfadjoint algebras.

2. Preliminaries. Throughout this paper, all Hilbert spaces discussed will be separable, all operators will be bounded, and all projections will be selfadjoint. Let $H$ be a Hilbert space. Then we write $L(H)$ for the collection of all bounded operators on $H, C(H)$ for the unit ball of $L(H)$ (i.e., the set of contractions on $H)$, and $P(H)$ for the lattice of projections on $H$. The latter two spaces are to be regarded as equipped with the strong operator topology and the Borel structure subordinate to it; this makes $P(H)$ and $C(H)$ into complete separable metric spaces.

Let $A$ be an operator on $H$. A projection $P$ is said to be invariant under $A$ 
if $P A P=A P$ and to reduce $A$ if $A P=P A$. (Usually one applies these terms to the range of $P$.) The lattice of all projections invariant under $A$ is denoted by Lat $A$. We write $A^{(n)}$ for an $n$-fold copy of $A$,i.e., the direct sum of $A$ with itself $n$ times; it acts on the Hilbert space $H^{(n)} \equiv H \oplus \cdots \oplus H$ ( $n$ copies).

Let. $S$ be a subset of $L(H)$. Then we write $S^{(n)}$ for $\left\{A^{(n)} \in L\left(H^{(n)}\right) \mid A \in S\right\}$, $S^{\prime}$ for the commutant of $S$ and $[S]$ for the strongly closed algebra generated by $S$. A projection is said to be invariant under $S$ if it is invariant under each operator in $S$. The lattice of all such projections is denoted by Lat $S$; dually we write Alg $Q$ for the algebra of all operators leaving each projection in a subset $Q$ of $P(H)$ invariant. Unless otherwise stated, the term algebra will always refer to an identity containing algebra of Hilbert space operators which is closed in the strong operator topology. It should perhaps be mentioned that the preference given to the strong operator topology in this paper is a matter of convenience only-saying an algebra is strongly closed is the same as saying it is weakly closedon the other hand, the fact that composition is strongly continuous on $C(H)$ will simplify some of our arguments. If an algebra is reduced by each of its invariant subspaces, it is called reductive and if it has no nontrivial reducing (invariant) subspaces it is called irreducible (transitive). Clearly, for selfadjoint or reductive algebras, irreducibility is equivalent to transitivity.

We now summarize some of the details of von Neumann's reduction theory. For the most part, we will follow [14], though it will prove convenient to make some small changes in the terminology. We begin by fixing, once and for all, a sequence of Hilbert spaces $h_{1} \subseteq h_{2} \subseteq \cdots \subseteq h_{\infty}$ with $h_{n}$ having dimension $n$ and $h_{\infty}$ spanned by the remaining $h_{n}$ 's. Next, suppose we have a partitioned measure space $\left(\Lambda, \mu,\left\{e_{n}\right\}\right)$. This means $\Lambda$ is a separable metric space, $\mu$ is (the completion of) a regular Borel measure on $\Lambda$, and $\left\{e_{n}\right\}_{n=1}^{n=\infty}$ is a Borel partition of $\Lambda$; we also assume that $\mu$ is $\sigma$-finite and $\Lambda$ is almost $\sigma$-compact. Then we form the associated direct integral Hilbert space $H=\int_{\Lambda}^{\oplus} h(\lambda) \mu(d \lambda)$. This consists of all (equivalence classes of) measurable functions $f$ from $\Lambda$ into $h_{\infty}$ such that $f(\lambda) \in$ $h(\lambda) \equiv h_{n}$ for $\lambda \in e_{n}$, and $\int_{\Lambda}\|f(\lambda)\|^{2} \mu(d \lambda)<\infty$. The element in $H$ represented by the function $\lambda \rightarrow f(\lambda)$ is denoted by $\int_{\Lambda}^{\oplus} f(\lambda) \mu(d \lambda)$.

An operator $A$ on $H$ is said to be decomposable if there exists a strongly $\mu$-measurable operator-valued function $A\left({ }^{\circ}\right)$ defined on $\Lambda$ such that $A(\lambda)$ is an operator on $h(\lambda)$ and for $f \in h, A f(\lambda)=A(\lambda) f(\lambda)$. We write $A=\int_{\Lambda}^{\oplus} A(\lambda) \mu(d \lambda)$ for the equivalence class corresponding to $A(\cdot)$. If $A(\lambda)$ is a scalar multiple of the identity on $h(\lambda)$ for almost all $\lambda$, then $A$ is called diagonal. The collection of all diagonal operators is called the diagonal algebra of $\Lambda$. It is an abelian von Neumann algebra.

The two basic results of reduction theory which will be used in the sequel 
are that (1) an operator acting on a direct integral of Hilbert spaces is decomposable if and only if it commutes with the corresponding diagonal algebra [16, p. 22] and (2) every abelian von Neumann algebra is (unitarily equivalent to) an essentially unique diagonal algebra [16, p. 19].

Finally, the following 'Principle of Measurable Choice' will be used repeatedly in our investigations. Von Neumann's original proof can be found in [12, $\S 16]$; a self-contained treatment can also be found in $\$ 1.4$ of [16].

Proposition 2.1. Suppose $\Lambda$ is a partitioned measure space, $Y$ is a complete metric space, and $E$ is a Borel relation contained in $\Lambda \times Y$. Then the domain of $E$ is a measurable of $\Lambda$. Furthermore, there exists a Borel function whose domain almost coincides with the domain of $E$ and whose graph is contained in $E$.

3. The decomposition. Fix a partitioned measure space $\Lambda$ and let $D$ be the corresponding diagonal algebra. Suppose we are given an algebra $\mathfrak{A}$ of decomposable operators. Then, of course, each operator $A \in \mathfrak{U}$ has a direct integral decomposition $A=\int_{\Lambda}^{\oplus} A(\lambda) \mu(d \lambda)$. What we wish to do here is define a corresponding decomposition for $\mathfrak{A}$. Mimicking the procedure in the selfadjoint case, we choose a countable generating set $\left\{A_{n}\right\}$ for $\mathfrak{U}$ and fix Borel representatives $\lambda \rightarrow A_{n}(\lambda)$ for their corresponding decompositions. For each $\lambda \in \Lambda$, we then define $\mathscr{U}(\lambda)$ to be the strongly closed algebra generated by the $\left\{A_{n}(\lambda)\right\}$. We write $\mathscr{U} \sim \int_{\Lambda}^{\oplus} \mathscr{U}(\lambda) \mu(d \lambda)$ and call this the decomposition of $\mathscr{A}$ with respect to $D$. In particular, we refer to subalgebras of $D^{\prime}$ (i.e., algebras of decomposable operators) as decomposable algebras.

In order that the preceding definitions be justified, it is necessary to show that up to a set of measure zero the algebras $\{\mathscr{A}(\lambda)\}$ are independent of the generating set $\left\{A_{n}\right\}$. In the selfadjoint case, this is done by appealing to the doublecommutant theorem-von Neumann algebras are completely determined by their commutants (cf. [16, pp. 46-48]). In the present situation, this tool is not available, and we use the following well-known property of strongly closed algebras in its place.

Proposition 3.1. Let $\mathfrak{U}$ be a strongly closed operator algebra. Then in order for an operator $A$ to belong to $\mathscr{A}$, it is necessary and sufficient that Lat $\mathfrak{U}^{(n)} \subseteq$ Lat $A^{(n)}$ for all $n$.

The following proposition is the basic result of this section.

Proposition 3.2. Let $\mathscr{U} \sim \int_{\Lambda}^{\oplus} \mathscr{U}(\lambda) \mu(d \lambda)$ be a decomposable algebra with $D$ the corresponding diagonal algebra. Suppose $A=\int_{\Lambda}^{\oplus} A(\lambda) \mu(d \lambda)$ is a decomposable operator. Then the set of $\lambda$ for which Lat $\mathcal{U}(\lambda) \subseteq$ Lat $A(\lambda)$ is measurable. Moreover, the following are equivalent. 
(1) Lat $[\mathscr{A}, D] \subseteq$ Lat $A$.

(2) Lat $\mathscr{U}(\lambda) \subseteq$ Lat $A(\lambda)$ for almost all $\lambda$.

Proof. For the sake of simplicity, we assume the Hilbert spaces $h(\lambda)$ all have the same dimension (and hence coincide with some fixed Hilbert space $h$ ), and that all the $\left\{A_{n}(\lambda)\right\}_{n, \lambda}$ are contractions. Trivial modifications adapt the proof to the more general case.

Set $E=\left\{(\lambda, Q) \in \Lambda \times P(h) \mid Q A_{n}(\lambda) Q=A_{n}(\lambda) Q\right.$ for each $n$, but $Q A(\lambda) Q$ $\neq A(\lambda) Q$ \}. Then $E$ is Borel (since composition is strongly continuous on $C(h)$ ), and the domain of $E$ consists of precisely those $\lambda \in \Lambda$ for which Lat $\mathscr{U}(\lambda) \mathbb{E}$ Lat $A(\lambda)$. Applying Proposition 2.1, we see that the initial conclusion of the proposition is established and we get a Borel function $P(\cdot)$ defined on almost all of the domain of $E$ such that $P(\lambda)$ is invariant under $\mathscr{U}(\lambda)$ but not under $A(\lambda)$. Set $P(\lambda)=0$ where it is not already defined and take $P=\int_{\Lambda}^{\oplus} P(\lambda) \mu(d \lambda)$. Then $P$ is a decomposable projection and hence $P \in \operatorname{Lat}[\mathscr{A}, D]$. On the other hand, $P$ will not belong to Lat $A$ unless the domain of $E$ has measure zero. This establishes the implication (1) $\Rightarrow(2)$.

To complete the proof, suppose (1) fails and let $P$ belong to Lat $[A, D]$ but not to Lat $A$. Then $P$ is decomposable and, for almost all $\lambda$, we have $P(\lambda) A_{n}(\lambda) P(\lambda)=A_{n}(\lambda) P(\lambda)$ for each $n$ whence $P(\lambda) \in$ Lat $2(\lambda)$. On the other hand, $P A P \neq A P$ so the set of $\lambda$ for which $P(\lambda) \notin$ Lat $A(\lambda)$ must have positive measure. Thus (2) fails and we have shown that $(2) \Rightarrow(1)$.

Proposition 3.3. Let $\mathcal{U} \sim \int_{\Lambda}^{\oplus} \mathcal{H}(\lambda) \mu(d \lambda)$ be a decomposable algebra with $D$ the corresponding diagonal algebra. Suppose $A=\int_{\Lambda}^{\oplus} A(\lambda) \mu(d \lambda)$ is a decomposable operator. Then the set of $\lambda$ for which $A(\lambda) \in \mathcal{A}(\lambda)$ is measurable. Moreover, the following are equivalent.

(1) $A \in[2, D]$.

(2) $A(\lambda) \in \mathscr{U}(\lambda)$ for almost all $\lambda$.

Proof. For each positive integer $k$, we can form $k$-fold copies of the objects $D, A$, and $\mathfrak{A}$. We regard these as being equipped with their corresponding direct integral decompositions; thus, for example, $\mathscr{U}^{(k)}(\lambda)$ is a weakly closed algebra of operators acting on the direct sum of $k$ copies of $h(\lambda)$, and is generated by the operators $\left\{A_{n}^{(k)}(\lambda)\right\}_{n=1}^{\infty}$.

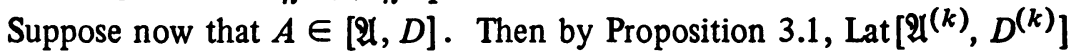
$\subseteq$ Lat $A^{(k)}$ for each $k$ and hence Proposition 3.2 gives Lat $\mathscr{U}^{(k)}(\lambda) \subseteq$ Lat $A^{(k)}(\lambda)$ for each $k$ for almost all $\lambda$. Thus a second application of Proposition 3.1 yields $A(\lambda) \in \mathscr{Q}(\lambda)$ for almost all $\lambda$ and we see that $(1) \Rightarrow(2)$. As the steps in this argument are all reversible, the Proposition is established.

As the following corollary shows, Proposition 3.3 settles all questions 
relating to the uniqueness of our decomposition. A family (or field as it is usually called) of algebras $\{\mathfrak{U}(\lambda)\}_{\lambda \in \Lambda}$ is attainable if there is some algebra $\mathscr{U}$ for which $\mathfrak{A} \sim \int_{\Lambda}^{\oplus} \mathfrak{A}(\lambda) \mu(d \lambda)$. The latter half of this paper will be concerned with the characterization of attainable fields.

COROllary 3.4. Let $D$ be a diagonal algebra.

(1) Every decomposable algebra has a unique decomposition with respect to $D$.

(2) Two decomposable algebras $\mathfrak{A}, \mathfrak{B}$ have the same decomposition iff $[\mathfrak{Q}, D]=[\mathfrak{B}, D]$.

(3) In particular, there is a one-to-one correspondence between attainable fields and algebras whose center contains $D$. The algebra corresponding to the field $\{\mathscr{A}(\lambda)\}$ consists of those decomposable operators $A=\int_{\Lambda}^{\oplus} A(\lambda) \mu(d \lambda)$ for which $A(\lambda) \in \mathscr{U}(\lambda)$ a.e.

Proof. Suppose first that $[\mathscr{A}, D]=[\mathscr{B}, D]$ and choose generating sets $\left\{A_{n}\right\}$ and $\left\{B_{n}\right\}$ for $\mathscr{U}$ and $\mathfrak{B}$, respectively. Since each $A_{n}$ belongs to $[\mathfrak{B}, D]$, the proposition gives $\left\{A_{n}(\lambda)\right\}_{n=1}^{\infty} \subseteq \mathfrak{B}(\lambda)$ for almost all $\lambda$. Thus $\mathfrak{H}(\lambda) \subseteq \mathfrak{B}(\lambda)$ a.e. and hence $\mathscr{Y}(\lambda)=\mathfrak{B}(\lambda)$ a.e. by symmetry. This establishes (1) and the 'if' part of (2). The remaining assertions of the corollary follow immediately from Proposition 3.3.

REMARK. If we restrict attention to selfadjoint algebras $\mathscr{U}$ and selfadjoint generating sets $\left\{A_{n}\right\}$, Proposition 3.3 becomes redundant and Corollary 3.4 follows immediately from Proposition 3.2; this is because von Neumann algebras are reflexive, i.e., $A \in \mathfrak{U}$ iff Lat $\mathfrak{A} \subseteq$ Lat $A$. In particular, Proposition 3.2 provides an alternative approach to the von Neumann decomposition.

EXAMPLE 3.5. The preceding corollary shows that the algebra $\mathscr{U}$ completely determines the algebras $\{\mathscr{L}(\lambda)\}$. The converse is not true in general, however. Indeed, let $\mathscr{A}$ be any algebra of operators on a Hilbert space $h$, set $h(1)=h(2)$ $=h$ and take $H=h(1) \oplus h(2)$. Then $\mathfrak{I}^{(2)}$ and $\mathfrak{U} \oplus \mathscr{U}$ have the same direct integral decompositions relative to the given decomposition of $H$.

We are now in a position to prove the extension of Mautner's result promised in the Introduction. A decomposition $\mathcal{A} \sim \int_{\Lambda}^{\oplus} \mathscr{A}(\lambda) \mu(d \lambda)$ of an algebra is said to be maximal if the corresponding diagonal algebra is maximal among the abelian von Neumann subalgebras of ' $\mathfrak{X}^{\prime}$. An application of Zorn's lemma shows that maximal decompositions exist for (algebras unitarily equivalent to) all strongly closed algebras.

THEOREM 3.6. The decomposition $\mathscr{A} \sim \int_{\Lambda}^{\oplus} \mathscr{U}(\lambda) \mu(d \lambda)$ is maximal if and only if almost all the algebras $\{2(\lambda)\}$ are irreducible.

Proof. Let $D$ be the diagonal algebra associated with the given 
decomposition. Let $P$ be any projection in $D^{\prime} \cap \mathscr{U}^{\prime}$. If almost all of the algebras are irreducible, then $P(\lambda)$ is trivial, so $P$ is a diagonal operator and hence must be in $D$. Therefore $D$ is maximal among the abelian von Neumann subalgebras of $\mathfrak{Q}^{\prime}$.

For the converse we assume $h(\lambda) \equiv h$ and choose a generating set $\left\{A_{n}\right\}$ for $\mathfrak{U}$ for which all the $\left\{A_{n}(\lambda)\right\}$ are contractions. Set $E=\{(\lambda, Q) \in \Lambda \times$ $P(h) \mid Q A_{n}(\lambda)=A_{n}(\lambda) Q$ for all $\left.n ; Q \neq 0, I\right\}$. Then $E$ is a Borel relation. Applying Proposition 2.1, we get a decomposable projection $P=\int_{\Lambda}^{\oplus} P(\lambda) \mu(\lambda)$ such that $P$ commutes with each $A_{n}$ and $P(\lambda)$ is nontrivial for almost all $\lambda$ in the domain of $E$. Thus $P \in[\mathscr{Q}, D]^{\prime}$ and by the maximality of $D$, we have $P \in D$, i.e., almost all of the $P(\lambda)$ are trivial. It follows that the domain of $E$ has measure zero and the proof is complete.

EXAMPLE 3.7. The only irreducible von Neumann algebra is the algebra of all operators. Thus, in a maximal decomposition $\mathscr{U} \sim \int \mathscr{U}(\lambda) \mu(d \lambda)$ of a selfadjoint algebra, we have $\mathscr{U}(\lambda)=L(h(\lambda)$ ) for almost all $\lambda$. A slight modification of Example 3.5 shows that such a decomposition also arises from some nonselfadjoint algebras. To see this, take $h(1)=h(2)=h$ and fix an invertible operator $S$ on $h$. Set $\mathscr{U}=\left\{A \oplus S A S^{-1} \mid A \in L(h)\right\}$. Then $\mathscr{Y}(1)=\mathfrak{Z}(2)=L(h)$, and since the adjoint of $A \oplus S A S^{-1}$ has $A^{*}$ as its first direct summand, $\mathscr{U}$ will only be selfadjoint when $\left(S A S^{-1}\right)^{*}=S A^{*} S^{-1}$ for all $A \in L(h)$. This, however, implies that $S^{*} S$ commutes with $L(h)$ and hence $\mathfrak{A}$ will fail to be selfadjoint when $S^{*} S$ is nonscalar.

EXAMPLE 3.8. The amount of information that can be lost in the decomposition of an algebra is graphically illustrated by [11, Theorem 1.5]. If $\mathscr{U} \sim$ $\int_{\Lambda}^{\oplus} \mathscr{A}(\lambda) \mu(d \lambda)$ is a maximal decomposition of any factor von Neumann algebra, then the underlying Hilbert spaces $\{h(\lambda)\}$ all coincide and hence $\mathscr{A}(\lambda) \equiv L(h)$. When $\mathscr{U}$ is of type $\mathbb{I}$, the algebras $\{\mathscr{U}(\lambda)\}$ fit together well; there will be a family of unitary operators $\{U(\lambda): h(\lambda) \rightarrow h\}$ such that $U(\lambda) A(\lambda)(U(\lambda))^{*}$ is essentially constant for each $A=. \int A(\lambda) \mu(d \lambda)$ in $\mathscr{A}$. No such relationship is possible when $\mathcal{Q}$ is of type II or III.

4. Applications to reductive algebras. The reductive algebra problem, introduced in [14], asks whether every reductive algebra is selfadjoint. A general discussion of reductive algebras and related topics can be found in [13]. The reductive algebra problem was introduced as a generalization of the transitive algebra problem (whether $L(H)$ is the only transitive algebra). The idea of the present section is to use the decomposition of $\$ 3$ to make a closer study of the relationship between these two problems.

THEOREM 4.1. Let $\mathscr{Q} \sim \int_{\Lambda}^{\oplus} \mathscr{\mathscr { A }}(\lambda) \mu(d \lambda)$ be a decomposition of a reductive algebra. Then almost all of $\{\Omega(\lambda)\}$ are reductive. In particular, if the decomposition is maximal, then almost all of the algebras $\{\Omega(\lambda)\}$ are transitive. 
Proof. Assume as usual that $h(\lambda) \equiv h$ and all of the $\left\{A_{n}(\lambda)\right\}_{n, \lambda}$ are contractions. Set $E=\left\{(\lambda, Q) \in \Lambda \times P(h) \mid Q\right.$ is invariant under all the $A_{n}$ 's but does not reduce them all $\}$. Then $E$ is Borel and the domain of $E$ consists of those $\lambda$ for which $\mathscr{U}(\lambda)$ is not reductive. Since $\mathfrak{U}$ is reductive, an application of Measurable Choice allows us to conclude that the domain of $E$ has measure zero. This establishes the first statement of the theorem. The second statement now follows by Theorem 3.6.

On first thought, it might seem that Theorem 4.1 establishes the general equivalence of the transitive and reductive algebra problems, but this is not the case. Indeed, knowing the algebras $\{\mathscr{A}(\lambda)\}$ does not give complete information about $\mathfrak{A}$ (see Example 3.5)-it is also necessary to know how the algebras $\mathscr{Q}(\lambda)$ 'fit together'; such is the case when we know that $\mathfrak{A}$ contains its invariant algebra (the algebra generated by the invariant projections of $\mathfrak{A}$ ). The following result uses this idea to reformulate the transitive algebra problem in terms of the reductive algebra problem.

COROLLARY 4.2. The following are equivalent.

(1) The only transitive algebra is the algebra of all operators.

(2) Every reductive algebra containing its invariant algebra is selfadjoint.

Proof. Assume (1) and let $\mathfrak{A} \sim \int_{\Lambda}^{\oplus} \mathfrak{A}(\lambda) \mu(d \lambda)$ be a maximal decomposition for an arbitrary reductive algebra. Then $\mathscr{U}(\lambda)=L(h(\lambda)$ ) for almost all $\lambda$. Thus if $A=\int_{\Lambda}^{\oplus} A(\lambda) \mu(d \lambda)$ belongs to $\mathfrak{A}$, then $A^{*}=\int_{\Lambda}^{\oplus} A^{*}(\lambda) \mu(d \lambda)$ will also belong to $\mathscr{Q}$ by virtue of Proposition 3.3 and we have $(1) \Rightarrow(2)$. The reverse implication is obvious as it is in each of the following corollaries.

In general, a reductive algebra will not contain its invariant algebra. Indeed, a factor cannot contain any nontrivial invariant projections, and the examination of how the algebras $\{\mathscr{U}(\lambda)\}$ arising from a maximal decomposition of type II factor 'fit together' is no simple task (cf. Example 3.8).

The preceding comments imply that there is little hope of using Theorem 4.1 to establish the global equivalence of the transitive and reductive algebra problems. Nevertheless, the decomposition of Theorem 4.1 can be fruitfully used to show the equivalence of certain 'natural parts' of these problems. Moreover, in certain cases where a particular case of the transitive algebra problem has been established, that result can be Iifted' to a corresponding result for reductive algebras. The first four of the corollaries below are examples of the former use of reduction theory, while the final corollary of the section is an example of the latter.

We begin with the result of J. Dyer, E. Pedersen and P. Porcelli announced in [4] and [5]. A short proof using the methods presented here appears in [3]. A single operator is said to be reductive (transitive) when the algebra it generates 
enjoys this property. The reductive operator problem asks whether every reductive operator is normal and this corollary shows it to be equivalent to the wellknown invariant subspace problem.

COROLLARY 4.3. The following are equivalent.

(1) Every transitive operator acts on a Hilbert space of dimension one.

(2) Every reductive operator is normal.

Proof. Assume (1) and let $A$ be a reductive operator. Choose a maximal decomposition $\mathfrak{A} \sim \int_{\Lambda}^{\oplus} \mathfrak{A}(\lambda) \mu(d \lambda)$ for the strongly closed algebra generated by $A$. By Theorem 4.1, almost all of the $\{A(\lambda)\}$ are transitive and hence the underlying Hilbert spaces $\{h(\lambda)\}$ are one-dimensional. It follows that $A$ is diagonal and we have the implication (1) $\Rightarrow(2)$.

REMARK. A reductive normal operator need not be selfadjoint. Indeed, if $N$ is any normal operator acting on a finite-dimensional space, then $N^{*} \in[N]$, so $N$ will be reductive.

It is easy to see that $L(h)$ is transitive and moreover that $L(h)$ is abelian only when $\operatorname{dim} h$ is 1 . It is natural to ask if this is a unique case.

COROLlARY 4.4. The following are equivalent.

(1) Every abelian transitive algebra acts on a Hilbert space of dimension one.

(2) Every abelian reductive algebra is selfadjoint.

Proof. As in the preceding corollaries, it suffices to establish the implication $(1) \Rightarrow(2)$. Thus let $\mathfrak{A}$ be an abelian reductive algebra, and choose a maximal decomposition $\mathscr{Q} \sim \int_{\Lambda}^{\oplus} \mathscr{U}(\lambda) \mu(d \lambda)$ for it. The algebras $\{\mathscr{A}(\lambda)\}$ all being transitive, we conclude that the underlying Hilbert spaces are one-dimensional. Thus $\mathfrak{A}$ consists of normal operators and consequently is selfadjoint by Sarason's Theorem [15].

A hyperinvariant subspace for an operator $A$ is a subspace invariant under $A^{\prime}$. Clearly only the trivial subspaces are hyperinvariant for a scalar multiple of the identity and it is an open question whether that is the case for any other operators. Thus the following corollary restates the so-called hyperinvariant subspace problem.

COROLLARY 4.5. The following are equivalent.

(1) Any operator without nontrivial hyperinvariant subspaces is scalar.

(2) If the commutant of a single operator is reductive, it (the commutant) is selfadjoint.

Proof. Assume (1) and let $A$ be an operator with $A^{\prime}$ reductive. Choose a maximal decomposition $\mathfrak{U} \sim \int_{\Lambda}^{\oplus} \mathfrak{A}(\lambda) \mu(d \lambda)$ for $\mathscr{U} \equiv A^{\prime}$. Now for almost all $\lambda$, 
$\mathscr{U}(\lambda) \subseteq[A(\lambda)]^{\prime}$ and $\mathscr{Y}(\lambda)$ is transitive. Thus almost all of the $\{A(\lambda)\}$ are scalar, i.e., $A$ is diagonal. The selfadjointness of $A^{\prime}$ now follows by Fuglede's Theorem.

The final two corollaries of this section depend on the following lemma, whose proof will be given in $\$ 5$; alternatively, one could modify the proof of Lemma 2 on page 46 of [16].

LEMMA 4.6. Let $\mathfrak{A} \sim \int_{\Lambda}^{\oplus} \mathfrak{A}(\lambda) \mu(d \lambda)$ be a decomposable algebra and suppose $D$ is the corresponding diagonal algebra. Then $[\mathfrak{A}, D]^{\prime} \sim \int_{\Lambda}^{\oplus}[\mathscr{A}(\lambda)]^{\prime} \mu(d \lambda)$.

COROLlary 4.7. The following are equivalent.

(1) The only transitive algebra containing its commutant is the algebra of all operators.

(2) Any reductive algebra containing its commutant must be selfadjoint.

Proof. Assume (1) and let $\mathscr{Q} \sim \int_{\Lambda}^{\oplus} \mathscr{A}(\lambda) \mu(d \lambda)$ be a maximal decomposition for a reductive algebra containing its commutant. By the lemma, $\mathfrak{A}^{\prime} \sim$ $\int_{\Lambda}^{\oplus}[\mathscr{U}(\lambda)]^{\prime} \mu(d \lambda)$ and hence $[\mathfrak{A}(\lambda)]^{\prime} \subseteq \mathfrak{U}(\lambda)$ for almost all $\lambda$ by Corollary 3.4 . Thus $\mathscr{\mathscr { C }}(\lambda)=L(h(\lambda))$ for almost all $\lambda$, and a second application of Corollary 3.4 yields $\mathfrak{Q}^{*} \subseteq \mathfrak{A}$.

Our final application of Theorem 4.1 concerns a 'lifting' of a transitive algebra result to reductive algebras. A von Neumann algebra is called a m.a.s.a. (maximal abelian selfadjoint algebra) if it coincides with its commutant. It follows from Lemma 4.6 that if a decomposable algebra contains a m.a.s.a., then so will almost all of its direct integrands. The following result is originally due to P. Rosenthal and H. Radjavi [14] where their proof is independent of the corresponding transitive algebra result.

COROLLARY 4.8. Every reductive algebra containing a m.a.s.a. is selfadjoint.

Proof. Let $\mathfrak{A} \sim \int_{\Lambda}^{\oplus} \mathfrak{Q}(\lambda) \mu(d \lambda)$ be a maximal decomposition of a reductive algebra containing a m.a.s.a. Then for almost all $\lambda, \mathfrak{I}(\lambda)$ is a transitive algebra containing a m.a.s.a, so $\mathfrak{U}(\lambda)=L(h(\lambda)$ ) by Theorem 3.3 of [1]. Thus $\mathfrak{A}=$ $[Q, D]$ is selfadjoint by Corollary 3.4 .

5. Attainable fields of algebras. Let $\Lambda$ be a partitioned measure space and $H=\int_{\Lambda}^{\oplus} h(\lambda) \mu(d \lambda)$, the associated direct integral of Hilbert spaces. For the sake of simplicity, we assume throughout this section that $h(\lambda) \equiv h$. In $\S 3$, starting with an algebra $\mathscr{A} \subseteq L(H)$, we constructed a field $\{\mathscr{H}(\lambda)\}_{\lambda \in \Lambda}$ of algebras. We now wish to ask when this process is reversible, i.e., starting with a field $\{\mathfrak{A}(\lambda)\}_{\lambda \in \Lambda}$ of algebras, when does there exist an algebra $\mathfrak{U} \subseteq L(H)$ for which $\mathfrak{A}$ $\sim \int_{\Lambda}^{\oplus} \mathscr{U}(\lambda) \mu(d \lambda)$ ? We will call the field $\{\mathscr{U}(\lambda)\}_{\lambda \in \Lambda}$ attainable tf this is the case. 
Of course $\$ 3$ gives us a characterization of attainable fields: $\{\mathfrak{Z}(\lambda)\}_{\lambda \in \Lambda}$ is attainable iff there exists a sequence $\left\{A_{n}\right\}_{n=1}^{\infty}$ of decomposable operators such that for each $\lambda, \mathfrak{M}(\lambda)$ is the algebra generated by $\left\{A_{n}(\lambda)\right\}_{n=1}^{\infty}$. This, however, is not very satisfying, and as pointed out by Effros [6] in the selfadjoint case, should be regarded as unnatural.

In this section, we will equip the collection of subalgebras of $L(h)$ with a Borel structure and show that the family $\{\mathscr{U}(\lambda)\}$ is attainable iff the function: $\lambda \mapsto \mathfrak{A}(\lambda)$ is measurable.

We begin our program by summarizing some of the terminology and results connected with measurable relations. The basic reference for this material is Himmelberg [7]. Throughout our discussion, $Y$ will be a complete separable metric space and $(\Lambda, \mu)$ will be a complete $\sigma$-finite measure space. The collection of closed nonempty subsets of $Y$ will be denoted by $2^{Y}$ and if $B$ is a subset of $Y$, we use the notation $\langle B\rangle$ for the collection $\left\{A \in 2^{Y} \mid A\right.$ is not disjoint from $\left.B\right\}$.

A function $F: \Lambda \rightarrow 2^{Y}$ is called a multifunction with closed values. Note that $F$ may also be viewed as a subset of $\Lambda \times Y$. A measurable selector for $F$ is a measurable function $\phi: \Lambda \rightarrow Y$ such that $\phi(\lambda) \in F(\lambda)$ for each $\lambda \in \Lambda$. The following proposition contains all the facts we need from [7].

Proposition 5.1. Let $F: \Lambda \rightarrow 2^{Y}$ be a multifunction with closed values. Then the following are equivalent:

(1) $F^{-1}(\langle B\rangle)$ is measurable for each closed $B \subseteq Y$.

(2) $F^{-1}(\langle B\rangle)$ is measurable for each open $B \subseteq Y$.

(3) $F^{-1}(\langle B\rangle)$ is measurable for each Borel $B \subseteq Y$.

(4) $F$ is a measurable subset of $\Lambda \times Y$.

(5) $F$ has a countable dense subset of measurable selectors.

Proof. The mutual equivalence of the first four conditions is Himmelberg's Theorem 3.5 (iii). Condition (5) means that there is a sequence $\left\{\phi_{n}\right\}$ of measurable selectors for $F$ such that $\left\{\phi_{n}(\lambda)\right\}_{n}$ is dense in $F(\lambda)$ for each $\lambda \in \Lambda$. Theorem 5.6 of [7] shows that (2) is equivalent to (5).

We will adopt condition (1) as the definition of measurability for the multifunction $F$. Stated somewhat differently, if we equip $2^{Y}$ with the $\sigma$-algebra structure generated by the hypersets $\langle B\rangle$ obtained as $B$ ranges through the closed subsets of $Y$, then $F$ is measurable precisely when it is measurable as a function from $\Lambda \rightarrow 2^{Y}$.

REMARK. Each of the first three conditions of the proposition induces a $\sigma$-algebra structure on $2^{Y}$. They do not always coincide. It is only because the domain measure space $(\Lambda, \mu)$ is complete that they give rise to the same measurable multifunctions. For more exact relationships under varying hypotheses, the reader is referred to [7]. 
Now let $h$ be a fixed Hilbert space. If $\mathfrak{A}$ is a strongly closed subalgebra of $L(h)$, then the unit ball of $\mathfrak{A}$ is a closed subset of $C(h)$; moreover, distinct algebras have distinct unit balls. Thus the collection of strongly closed subalgebras of $L(h)$ may be identified with a subset of $2^{C(h)}$. We will make this identification throughout the sequel.

The following is half of the main result in this section.

Proposition 5.2. If $F: \lambda \mapsto \mathscr{\Re}(\lambda)$ is a measurable field of subalgebras of $L(h)$, then it is attainable.

Proof. This is an immediate consequence of Proposition 5.1. Indeed if $F$ is measurable, then there exists a countable dense family $\lambda \mapsto A_{n}(\lambda)$ of selectors for $F$. Thus for each $\lambda \in \Lambda$, the set $\left\{A_{n}(\lambda)\right\}_{n=1}^{\infty}$ is dense in the unit ball of $\mathscr{U}(\lambda)$ and a fortiori the $\left\{A_{n}(\lambda)\right\}$ generate $\mathscr{U}(\lambda)$.

If we restrict attention to selfadjoint algebras, then the argument of the preceding paragraph is reversible. The proof, due to Effros [6], proceeds as follows: Assume $\mathfrak{Q} \sim \int_{\Lambda}^{\oplus} \mathfrak{A}(\lambda) \mu(d \lambda)$ with $\mathscr{U}$ and $\{\mathfrak{U}(\lambda)\}$ all selfadjoint. Let $\left\{A_{n}\right\}_{n=1}^{\infty}$ be a selfadjoint generating set for $\mathscr{U}$ which is an algebra over the rational numbers and is closed under adjunction and multiplication by $i$. Then the $\left\{A_{n}(\lambda)\right\}_{n}$ are dense in $\mathscr{U}(\lambda)$ for almost all $\lambda$. By the Kaplansky Density Theorem, the intersection of $\left\{A_{n}(\lambda)\right\}_{n}$ with $C(h)$ will also be dense in $\mathscr{U}(\lambda) \cap C(h)$. Setting

$$
\phi_{n}(\lambda)= \begin{cases}A_{n}(\lambda) & \text { if } A_{n}(\lambda) \text { is a contraction } \\ I & \text { otherwise }\end{cases}
$$

we have a countable dense set of selectors for $F$ and thus $F$ is measurable by Proposition 5.1.

Since nonselfadjoint algebras do not always satisfy the Kaplansky Theorem, a different argument is needed for the converse of Proposition 5.2.

LEMMA 5.3. Let $F: \lambda \mapsto \mathscr{U}(\lambda)$ be an attainable field of algebras. Then Lat $F: \lambda \mapsto$ Lat $\mathscr{I}(\lambda)$ and $F^{\prime}: \lambda \mapsto[\mathscr{H}(\lambda)]^{\prime}$ are measurable multifunctions.

Proof. Let $\lambda \mapsto A_{n}(\lambda)$ be measurable fields of operators generating the $\{\mathscr{R}(\lambda)\}$. Then Lat $F=\{(\lambda, P) \mid P$ is a selfadjoint projection invariant under each $\left.A_{n}(\lambda), n=1,2, \ldots\right\}$. Since $(\lambda, P) \mapsto P A_{n}(\lambda) P-A_{n}(\lambda) P$ is measurable, Lat $F$ is a measurable subset of $\Lambda \times C(h)$. The argument for $F^{\prime}$ is similar.

We are now in a position to give an easy proof of a result used in the previous section.

Proof of Lemma 4.6. Let $\mathscr{U} \sim \int_{\Lambda}^{\oplus} \mathscr{U}(\lambda) \mu(d \lambda)$. Then the multifunction

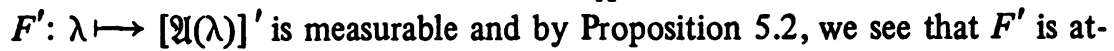
tainable. Corollary 3.4 tells us there is a unique diagonal containing algebra $\mathfrak{B}$ 
for which $\mathfrak{B} \sim \int_{\Lambda}^{\oplus}[\mathcal{A}(\lambda)]^{\prime} \mu(d \lambda)$. Moreover, the operators in $\mathscr{B}$ are precisely those decomposable operators $B=\int_{\Lambda}^{\oplus} B(\lambda) \mu(d \lambda)$ for which $B(\lambda) \in[\mathscr{R}(\lambda)]^{\prime}$ a.e. This means $B$ commutes with $D$ and with each $A_{n}$. Thus $\mathscr{B}=[\mathfrak{A}, D]^{\prime}$.

Let $S$ be a subset of $L(h)$ and $n$ a positive integer. An operator $A \in L(h)$ is said to belong to the $n$-closure of $S$ if Lat $S^{(n)} \subseteq$ Lat $A^{(n)}$. The $n$-closure of $S$ is a strongly closed subalgebra of $L(h)$ and will be denoted by $\mathrm{Cl}_{n}(S)$. By virtue of Proposition 3.1, the strongly closed algebra generated by $S$ is $\bigcap_{n=1}^{\infty} \mathrm{Cl}_{n}(S)$.

Lemma 5.4. Let $F: \lambda \mapsto \mathscr{A}(\lambda)$ be an attainable field of algebras. Then the multifunction $\mathrm{Cl}_{n}(F): \lambda \mapsto n$-closure of $\mathcal{Q}(\lambda)$ is measurable.

PRoof. Since the field $\lambda \mapsto \mathscr{I}^{(n)}(\lambda)$ is attainable, Lemma 5.3 shows that the multifunction: $\lambda \mapsto$ Lat $\mathfrak{H}^{(n)}(\lambda)$ is measurable. Let $\left\{P_{k}\right\}_{k=1}^{\infty}$ be a countable dense set of measurable selectors for this multifunction. Then $\mathrm{Cl}_{n}(F)=\{(\lambda, A)$ $\in \Lambda \times C(h) \mid$ each $P_{k}(\lambda)$ is invariant under $\left.A^{(n)}\right\}$ is a measurable subset of $\Lambda \times$ $C(h)$.

Now we can prove the converse of Proposition 5.2.

THEOREM 5.5. Let $F: \lambda \mapsto \mathscr{Q}(\lambda)$ be a field of subalgebras of $L(h)$. Then the following are equivalent:

(1) $F$ is attainable.

(2) $F$ is measurable.

Proof. We need only establish the implication $(1) \Rightarrow(2)$. Thus let $F$ : $\lambda \mapsto \mathscr{A}(\lambda)$ be an attainable field of algebras. By the previous lemma $\mathrm{Cl}_{n}(F)$ is a measurable subset of $\Lambda \times C(h)$. Therefore, so is $\bigcap_{n=1}^{\infty} \mathrm{Cl}_{n}(F)$. But $F=$ $\bigcap_{n=1}^{\infty} \mathrm{Cl}_{n}(F)$ as was noted before Lemma 5.4.

We close with an application of Theorem 5.5 to reflexive algebras. Recall that $\mathfrak{U}$ is said to be reflexive if $\mathfrak{U}=\mathrm{Alg}$ Lat $\mathfrak{U}$. Note that $\mathrm{Alg}$ Lat $\mathfrak{U}$ is the 1 closure of $\mathcal{Q}$.

Proposition 5.6. Let $\mathscr{U} \sim \int_{\Lambda}^{\oplus} \mathscr{\mathscr { A }}(\lambda) \mu(d \lambda)$ be a decomposable algebra corresponding to the diagonal algebra $D$. Then Alg Lat [U, D] $\int_{\Lambda}^{\oplus} \operatorname{Alg} \operatorname{Lat}[\mathcal{H}(\lambda)] \mu(d \lambda)$. In particular, reflexivity of $[\mathscr{A}, D]$ is equivalent to the reflexivity of almost all of the $\{\mathfrak{A}(\lambda)\}$.

Proof. Lemma 5.4 shows that the field $\{\text { Alg Lat } \mathscr{R}(\lambda)\}_{\lambda \in \Lambda}$ is attainable and hence there is a unique diagonal-containing algebra $\mathscr{B} \sim$ $\int_{\Lambda}^{\oplus} \operatorname{Alg}$ Lat $\mathscr{I}(\lambda) \mu(d \lambda)$. Suppose $B \in \mathfrak{B}$. Then $B(\lambda) \in \operatorname{Alg}$ Lat $\mathscr{U}(\lambda)$, i.e., Lat $\mathscr{U}(\lambda) \subseteq$ Lat $B(\lambda)$ a.e. Thus Lat $[\mathfrak{A}, D] \subseteq$ Lat $B$ by Proposition 3.2 so $B \in$ $\mathrm{Alg}$ Lat $[\mathfrak{A}, D]$. Since this chain of reasoning is reversible, we conclude that $\mathscr{B}=$ Alg Lat $[\mathscr{\vartheta}, D]$ and the first statement of the proposition is established. The second statement is now a consequence of Corollary 3.4(3): $\$$ will coincide with $[\mathfrak{R}, D]$ iff $\mathscr{R}(\lambda)=\mathscr{B}(\lambda)$ a.e. 
The following two examples illustrate that the role of $D$ in the preceding proposition is essential: in general there is no relationship between the reflexivity of $\mathscr{U}$ and that of $[\mathscr{U}, D]$.

EXAMPLE 5.7. Let

and take

$$
\mathscr{U}=\left\{\left[\begin{array}{ll}
a & b \\
0 & a
\end{array}\right] \oplus\left[\begin{array}{ll}
a & b \\
0 & a
\end{array}\right] \in M_{4} \mid a, b \in \mathrm{C}\right\}
$$

$$
D=\left\{\left[\begin{array}{ll}
\lambda & 0 \\
0 & \lambda
\end{array}\right] \oplus\left[\begin{array}{ll}
\mu & 0 \\
0 & \mu
\end{array}\right] \in M_{4} \mid \lambda, \mu \in \mathrm{C}\right\} .
$$

Then $\mathfrak{U}$ is reflexive but $[\mathfrak{A}, D]$ is not reflexive. Indeed, the first assertion is clear and the second assertion follows from Proposition 5.6 since $\left\{\left[\begin{array}{ll}a & b \\ 0 & a\end{array}\right] \in\right.$ $\left.M_{2} \mid a, b \in \mathrm{C}\right\}$ is nonreflexive.

The phenomenon of the previous example cannot occur for reductive algebras. Saying a reductive algebra is reflexive is the same as saying it is selfadjoint, and clearly if $\mathfrak{A}$ is selfadjoint, so is [थ, $D$ ]. On the other hand, it is not known whether reductive algebras can exhibit the behavior of the following example; the discussion surrounding Corollary 4.2 should be interpreted as saying that the possibility of this type of behavior is the 'obstruction' to the equivalence of the transitive and reductive algebra problems.

EXAMPLE 5.8. Let

$$
\left.\mathfrak{U}=\left\{\begin{array}{llll}
a & 0 & 0 & 0 \\
0 & a & b & c \\
0 & 0 & a & d \\
0 & 0 & 0 & a
\end{array}\right] \oplus\left[\begin{array}{lll}
a & e & 0 \\
0 & a & 0 \\
0 & 0 & a
\end{array}\right] \in M_{4} \oplus M_{3} \mid \begin{array}{l}
a, b, c, d, e \text { are complex } \\
\text { numbers and } b+d+e=0
\end{array}\right\}
$$

and take $D=\left\{\lambda \boxplus \oplus I \in M_{4} \oplus M_{3} \mid \lambda, \mu \in C\right\}$.

Claim $1 . \mathfrak{A}$ is not reflexive.

Set

$$
A_{0}=0 \oplus\left[\begin{array}{lll}
0 & 1 & 0 \\
0 & 0 & 0 \\
0 & 0 & 0
\end{array}\right] \in M_{4} \oplus M_{3}
$$

Then $A_{0} \notin \mathscr{U}$. We will show $A_{0} x \in \mathscr{U} x$ for each vector $x \in \mathrm{C}_{7}$. This implies Lat $\mathfrak{A} \subseteq$ Lat $A_{0}$ and hence our claim will be established. Thus pick $x=$ $\left(x_{1} \cdots x_{7}\right) \in \mathbf{C}_{7}$.

Case (a). $x_{3} \neq 0$ but $x_{4}=0$. Then set 


$$
A=\left[\begin{array}{rrrr}
0 & 0 & 0 & 0 \\
0 & 0 & 0 & 0 \\
0 & 0 & 0 & -1 \\
0 & 0 & 0 & 0
\end{array}\right] \oplus\left[\begin{array}{lll}
0 & 1 & 0 \\
0 & 0 & 0 \\
0 & 0 & 0
\end{array}\right]
$$

Then $A \in \mathfrak{A}$ and $A_{0} x=A x$.

Case (b). $x_{3}=0$ or $x_{4} \neq 0$. Then the equation $-x_{3}+\lambda x_{4}=0$ has at least one solution $\lambda$. We set

$$
A=\left[\begin{array}{rrrr}
0 & 0 & 0 & 0 \\
0 & 0 & -1 & \lambda \\
0 & 0 & 0 & 0 \\
0 & 0 & 0 & 0
\end{array}\right] \oplus\left[\begin{array}{lll}
0 & 1 & 0 \\
0 & 0 & 0 \\
0 & 0 & 0
\end{array}\right]
$$

and we have $A_{0} x=A x \in \mathscr{I} x$ as desired. (This construction is related to Example 3.2 of [2] .)

Claim 2. [थ, D] is reflexive.

By Proposition 5.6, it suffices to show the algebras

$$
\mathfrak{q}_{1}=\left\{\left[\begin{array}{cccc}
a & 0 & 0 & 0 \\
0 & a & b & c \\
0 & 0 & a & d \\
0 & 0 & 0 & a
\end{array}\right] \in M_{4}\right\} \text { and } \mathscr{V}_{2}=\left\{\left[\begin{array}{lll}
a & e & 0 \\
0 & a & 0 \\
0 & 0 & a
\end{array}\right] \in M_{3}\right\}
$$

are both reflexive. We will deal with $\mathfrak{A}_{2}$ here; the proof for $\mathfrak{A}_{1}$ is similar. Thus suppose $A \in M_{3}$ leaves every subspace in Lat $\mathscr{I}_{2}$ invariant. Then clearly $A$ is upper triangular and has $e_{3}$ as an eigenvector. Thus $A$ is of the form

$$
\left[\begin{array}{lll}
a_{1} & e & 0 \\
0 & a_{2} & 0 \\
0 & 0 & a_{3}
\end{array}\right]
$$

Moreover, consideration of the subspace 


$$
\left\{(y, x, x) \in \mathrm{C}_{3} \mid y \in \mathrm{C}, x \in \mathrm{C}\right\}
$$

shows $a_{2}=a_{3}$ while consideration of $\left\{(x, 0, x) \in \mathrm{C}_{3}\right\}$ gives $a_{1}=a_{3}$. Thus $A \in$ $\mathscr{U}_{2}$ and $\mathscr{H}_{2}$ is reflexive.

\section{REFERENCES}

1. W. B. Arveson, $A$ density theorem for operator algebras, Duke Math. J. 34 (1967), 635-647. MR $36 \# 4345$.

2. E. A. Azoff, K-reflexivity in finite dimensional spaces, Duke Math. J. 40 (1973), 821-830. MR 48 \#9415.

3. E. A. Azoff and F. Gilfeather, Measurable choice and the invariant subspace prob. lem, Bull. Amer. Math. Soc. 80 (1974), 893-895. MR 50 \#14276.

4. J. A. Dyer, E. A. Pedersen and P. Porcelli, An equivalent formulation of the invariant subspace conjecture, Bull. Amer. Math. Soc. 78 (1972), 1020-1023. MR 46 \#6068.

5. J. A. Dyer and P. Porcelli, Concerning the invariant subspace problem, Notices Amer. Math. Soc. 17 (1970), 788. Abstract \#677-47-4.

6. E. G. Effros, The Borel space of von Neumann algebras on a separable Hilbert space, Pacific J. Math. 15 (1965), 1153-1164. MR 32 \#2923.

7. C. Himmelberg, Measurable selections, Fund. Math. 87 (1975), 53-72.

8. K. Kuratowski, Topology, Vols. I, II, revised and augmented, PWN, Warsaw; Academic Press, New York, 1968. MR 36 \#840; 41 \#4467.

9. K. Kuratowski and C. Ryll-Nardzewski, A general theorem on selectors, Bull. Acad. Polon. Sci. Sér. Sci. Math. Astronom. Phys. 13 (1965), 397-403. MR 32 \#6421.

10. O. Maréchal, Topologie et structure borélienne sur l'ensemble des algèbres de von Neumann, C. R. Acad. Sci. Paris Sér. A-B 276 (1973), A847-A850. MR 47 \#5611.

11. F. I. Mautner, Unitary representations of locally compact groups. I, Ann. of Math. (2) 51 (1950), 1-25. MR 11, 324.

12. J. von Neumann, On rings of operators. Reduction theory, Ann. of Math. (2) 50 (1949), 401-485. MR 10, 584.

13. H. Radjavi and P. Rosenthal, Invariant subspaces, Springer-Verlag, Berlin, 1973.

14. - A sufficient condition that an operator algebra be self-adjoint, Canad. J. Math. 23 (1971), 588-597.

15. D. Sarason, Invariant subspaces and unstarred operator algebras, Pacific J. Math. 17 (1966), 511-517. MR 33 \#590. \#547.

16. J. T. Schwartz, $W^{*}$-algebras, Gordon and Breach, New York, 1967. MR 38

DEPARTMENT OF MATHEMATICS, UNIVERSITY OF IOWA, IOWA CITY, IOWA 52242

DEPARTMENT OF MATHEMATICS, UNIVERSITY OF TORONTO, TORONTO (M5S 1A7) ONTARIO, CANADA (Current address of C. K. Fong)

DEPARTMENT OF MATHEMATICS, UNIVERSITY OF NEBRASKA, LINCOLN, NEBRASKA 68508 (Current address of F. Gilfeather)

Current address (E. A. Azoff): Department of Mathematics, University of Georgia, Athens, Georgia 30602 